\title{
Biomechanical analysis to characterize the impact of knee osteoarthritis on hip, knee, and ankle kinematics
}

\author{
Neila Mezghani*1,2, Delphine Billard ${ }^{2}$, Youssef Ouakrim ${ }^{1}$, Alexandre Fuentes ${ }^{2}$, Nicola Hagemeister ${ }^{2}$, Jacques A de \\ Guise $^{2}$ \\ ${ }^{1}$ LICEF research center, TÉLUQ university, Montreal, QC, Canada \\ ${ }^{2}$ Laboratoire de recherche en imagerie et orthopédie, École de technologie supérieur, Centre de recherche du CHUM, Montréal, \\ QC, Canada
}

Received: December 12, 2016

Accepted: May 1, 2017

Online Published: May 10, 2017

DOI: $10.5430 /$ jbei.v3n2p36

URL: https://doi.org/10.5430/jbei.v3n2p36

\begin{abstract}
Background: Numerous studies use a biomechanical assessment to evaluate joint function in knee pathologies such as osteoarthritis. However, most of them focus only on the knee and the consequences of the pathology on other lower limb joints are poorly documented. The objective of this study is to analyze the impact of knee osteoarthritis on ipsilateral hip, knee and ankle joint during gait.

Methods: Three-dimension (3D) angular kinematic patterns of the three joints were analyzed on 32 patients diagnosed with knee osteoarthritis (OA) and a control group of 15 asymptomatic subjects (AS). Kinematic data was captured during treadmill gait trials at a self-selected comfortable speed. Analysis of covariance (ANCOVA) was performed on selected points of interest from 3D kinematic patterns of the hip, knee and ankle joints to compare both groups. The significance level was set at $p=.05$.

Results: Gait 3D kinematic gait patterns of OA patients revealed significant differences with those of AS subjects at the three joints.

Conclusions: Results suggest that patients with knee osteoarthritis also present alterations in hip and ankle kinematic during gait that should be considered when tailoring conservative treatments.
\end{abstract}

Key Words: Knee osteoarthritis, Biomechanical analysis, Kinematics, Hip, Knee, Ankle

\section{INTRODUCTION}

Knee osteoarthritis (OA) is a musculoskeletal disorder involving the degeneration of the knee cartilage, which leads to pain and functional limitations. Its prevalence has been estimated by the World Health Organisation to be around $10 \%$ of the adult population in industrialized countries. ${ }^{[1]}$ With the population ageing and the prevalence of obesity increasing, the number of people who will suffer from knee osteoarthritis is anticipated to increase in the next decades.
Conservative management through physical therapy, exercises, weight management and education allows to improves patient quality of life, and reduce pain and limitations. ${ }^{[2-4]}$ However, OA is still one of the most important chronic diseases in the use of health services implicating high direct and indirect costs. Hunter et al. suggested that placing more attention to the role of mechanical factors in knee OA progression could help find ways to reduce public health impact. ${ }^{[3]}$

Previous studies have shown that mechanical factors, such

\footnotetext{
*Correspondence: Neila Mezghani; Email: Neila.mezghani@teluq.ca; Address: Centre de recherche LICEF de la TÉLUQ, 5800 Rue Saint-Denis, Montreal, QC H2S 3L4, Canada.
} 
as misalignment are important risk factors in the progression of the knee OA. In fact, static parameters such as femorotibial alignment may have less influence over medial OA progression than dynamic factors such as the varus thrust ${ }^{[5]}$ supporting the need for biomechanical assessments. Knee osteoarthritis to affect the dynamic function of the knee in all three planes of movement. ${ }^{[6]}$ In fact, previous studies have essentially focused their biomechanical assessment on the knee joint and there is limited information on the impact of knee OA on the ipsilateral hip and ankle joints. This type of information would be valuable since it was suggested that physiotherapy treatment should not only target knee musculature, but also the hip in order to reduce external knee adduction moment, a surrogate of knee joint loading. ${ }^{[7]}$ They also demonstrated that strengthening hip muscles reduced symptoms and improved the function of the OA patient's knee. ${ }^{[8]}$

We found one study looking at the link between biomechanical changes at the knee, the hip, and the ankle in 2008. ${ }^{[9]}$ The authors measured the knee and hip moments using an inverse dynamic model after gait analysis with skin markers. They concluded that, with increasing severity of knee OA, knee flexion angles decreased, early stance knee extension moments decreased, stance phase hip internal rotation moments decreased and peak ankle dorsiflexion moments decreased. Ko et al. verified the hypothesis that individuals with symptomatic knee OA had a similar or higher Mechanical work expenditures (MWE) and higher range of motion at the ankle than asymptomatic individuals. ${ }^{[10]}$ Farrokhi et al. showed that OA patients with self-reported knee instability had reduced contributions from the hip extensors and ankle plantar flexors during gait. ${ }^{[11]}$ Such studies provide valuable quantitative information, but are difficult to include in clinical practice because of the need of very sophisticated technology and inverse dynamic models to provide moment measurements in the three planes.

It is now recognized that three-dimensional kinematic evaluations are helpful in performing a functional assessment of the joint in a clinical setting. By using a non-invasive attachment system, designed to reduce skin movement artifacts, it is now possible to have access to three dimensional measures of knee angles during gait with acceptable accuracy and reproducibility. This has recently been performed on an OA population. ${ }^{[12]}$ The authors showed that OA patients on a waiting list for TKA displayed reduced knee extension during stance phase, reduced knee flexion during push-off, increased knee adduction angle, and reduced tibial rotation during midstance phase. This study did not investigate the relationship of these changes with hip and ankle kinematics.
To our knowledge, angular kinematic patterns of all three adjacent joints of the lower limb, and their relationship in the cases of knee OA have not yet been investigated. Advanced knowledge on these relationships would provide the scientific rational for multi-joint approaches of knee OA treatments, as suggested in previous studies, ${ }^{[7,8]}$ and could be included, on the longer term, into clinical management of the disease.

Our hypothesis is that the kinematic modifications that take place at the knee, following onset of OA, will have an impact on hip and ankle kinematics, modifying in turn kinematic patterns of these joints. The purpose of this study was to analyze the impact of knee osteoarthritis on hip and ankle 3D angular kinematic patterns during gait.

\section{METHOD}

\subsection{Participants}

The biomechanical data were obtained during a gait analysis protocol from a previous studies. ${ }^{[13,14]}$ Two groups of participants were included in the study: 15 asymptomatic subjects formed the control group (AS) and 32 patients affected by clinically and radiologically confirmed femorotibial osteoarthritis formed the knee OA group (OA). AS subjects came from three other projects. Inclusion criteria were no history of knee pain and no previous lower lamb injury. For ethical reasons, these subjects did not have an X-ray. That is the reason why they are called asymptomatic and not "normal" or "healthy" subjects.

The mean biometric characteristics of the participants are presented in Table 1. All subjects gave their consent to participate, which was approved by institutional ethics committees.

Table 1. Participants' age and anthropomorphic characteristics

\begin{tabular}{lll}
\hline Characteristics & AS & OA \\
\hline Sample size & 15 & 32 \\
Age (mean years $\pm \mathrm{SD}$ ) & $66 \pm 7$ & $63 \pm 8$ \\
Height (mean m $\pm \mathrm{SD}$ ) & $1.619 \pm 0.090$ & $1.612 \pm 0.101$ \\
Body weight (mean kg $\pm \mathrm{SD})$ & $64.95 \pm 12.23$ & $81.39 \pm 17.65 *$ \\
Body mass index (mean kg/m $\left.{ }^{2} \pm \mathrm{SD}\right)$ & $24.69 \pm 3.71$ & $31.05 \pm 4.51$ \\
Percentage of women (number) & $67(10)$ & $72(23)$ \\
Walking velocity (mean m/s $\pm \mathrm{SD})$ & $0.85 \pm 0.20$ & $0.82 \pm 0.20$ \\
\hline
\end{tabular}

Note. Values are mean $\pm \mathrm{SD} . *$ Significant at $p<.002$.

\subsection{Data acquisition}

During the biomechanical evaluations, kinematics data were collected using a six-camera optoelectronic system (VICON 460, Oxford Metrics) during a 25-s walking trial, performed at a self-determined comfortable speed on a treadmill. Rigid bodies including four reflective markers were fixed at the pelvis using a sacro-illiac stabilization belt, at the knee on 
femoral and tibial parts of an exoskeleton (KneeKG, Emovi Inc), and on the foot over the navicular bone. To assess hip kinematics, we recorded the motion of the femoral tracker in regards to the pelvis tracker. For the knee, the KneeKG was used since it was designed to significantly reduce relative movement between skin and bone and it has been extensively validated in previous studies. ${ }^{[15,16]}$ It provides repeatable $\left(0.4^{\circ}-0.8^{\circ}\right.$ and $\left.0.8-2.2 \mathrm{~mm}\right)$ and reliable (intra-class coefficient of 0.88-0.94) measurements of knee kinematics. ${ }^{[16]}$ In order to measure ankle kinematics, additional reflective markers were fixed onto the lateral and medial malleoli, onto the calcaneus, and onto the distal phalanx of the second metatarsal.

Femoral and tibial coordinate systems were defined using a functional and postural approach (FP method). ${ }^{[15]}$ The hip joint centre (HJC) was defined by an optimization method during a leg circumduction movement; the knee joint centre (KJC) was defined by projecting the midpoint from the lateral and medial femoral epicondyles on a mean helical knee flexion-extension axis; and the ankle joint centre (AJC) corresponded to the midpoint between lateral and medial malleoli. ${ }^{[14]}$

\subsection{Data processing}

A data processing was performed as following. No filtering was applied as resampling and averaging were considered sufficient to remove noise artefacts caused by data acquisition. Gait cycles events were identified using ground reaction forces collected with two Kistler forces plates integrated into the treadmill (Adal, TECMACHINE, Medical development). The gait cycles were normalized $(1 \%-100 \%)$ between two successive foot contacts, which corresponded to instants when the magnitude of vertical ground reaction forces exceeded $2 \%$ of the participant's body weight.

For each subject, 15 representative gait cycles were finally selected and averaged. ${ }^{[17]}$ The representative curves that maximize the intra subject correlation index are averaged to obtain mean GCs per subject. A mean pattern on 100 points in each plane at each joint for each subject was obtained. Their variables were: at the hip adduction/abduction, extension/flexion and femoral external/internal rotation; at the knee adduction/abduction, flexion/extension and tibial external/internal rotation; at the ankle inversion/eversion, plantar flexion/dorsiflexion and external/internal rotation.

\subsection{Data analysis}

A preliminary analysis, ANOVA has been applied on biometric variables and on gait velocity. Table 1 demonstrated a significant difference in the weight between the two groups. OA participants were significantly heavier than AS ones $(p=$ .002 ), with $16 \mathrm{~kg}$ more on average. The weight was included as a covariate in subsequent statistical analyses to eliminate confounding effects.

Analysis of covariance (ANCOVA) was performed on points of interest on the 3D kinematics patterns in order to compare means between groups and identify local parameters allowing distinguishing between groups. The weight was included as a covariate in subsequent analyses of covariance.

Points of interest are angular kinematic parameters that are interesting for further biomechanical analysis. ${ }^{[18,19]}$ They are collected on each joint/plan signal at specific time frames. These angular variables are local maximums and minimums, average angular positions and angular changes on a gait subcycle, and range of motion. Points of interest were defined on gait sub-cycles identified by the specific time cycles. The parameters chosen to be extracted were based on variables routinely assessed in clinical biomechanical studies of knee OA populations as proposed in the literature. ${ }^{[12,13,17]}$ These are summarized in Table 2, with acronyms chosen to identify events.

Table 2. Terms used to identify major events during the gait cycle ${ }^{[20]}$

\begin{tabular}{lllllllll}
\hline Events & $\begin{array}{l}\text { Loading } \\
\text { response }\end{array}$ & Mid-stance & $\begin{array}{l}\text { Terminal } \\
\text { stance }\end{array}$ & Pre-swing & Initial swing & Mid-swing & Terminal swing \\
\hline Acronyms & $\mathbf{T}_{\mathbf{0}}$ & $\mathbf{T}_{\mathbf{1}}$ & $\mathbf{T}_{\mathbf{2}}$ & $\mathbf{T}_{3}$ & $\mathbf{T}_{\mathbf{4}}$ & $\mathbf{T}_{5}$ & $\mathbf{T}_{\mathbf{6}}$ & $\mathbf{T}_{\mathbf{7}}$ \\
GC (\%) & 1 & 10 & 30 & 50 & 60 & 73 & 87 & 100 \\
\hline
\end{tabular}

Note. GC: Gait Cycle percentage.

The angular kinematic parameters values were extracted for all subjects on MatlabR2015a (The Mathworks, USA) and ANCOVA was performed on SPSS 20.0 (Statistical Package for the Social Sciences). The significance level was set at $p$ $=.05$.

\section{RESULTS}

AS and OA average kinematic patterns of the hip, knee and ankle in frontal, sagittal and transversal planes are presented in Figure 1. Only significant results are detailed in Table 3 and commented hereafter. 
At the hip, OA patients showed a lower adduction movement group kept a position quite stable in abduction $\left(\Delta \theta_{H F 5}\right.$ and during the stance phase $\left(\Delta \theta_{H F 1}, \Delta \theta_{H F 2}\right.$ and $\left.\Delta \theta_{H F 3}\right)$ and $\left.\Delta \theta_{H F 6}\right)$. No difference was found at the hip in the sagittal started the abduction movement later at pre-swing $\left(\Delta \theta_{H F 4}\right)$. plane between groups. In the transverse plane, OA subjects At the end of the gait cycle, AS group accomplished an ad- were less in external rotation of the hip than controls at preduction which continued at the next gait cycle while OA swing $\left(\theta_{H T 1}\right)$.

Table 3. Statistical analysis of hip, knee and ankle kinematic parameters

\begin{tabular}{|c|c|c|c|c|c|}
\hline & & AS & $\mathbf{O A}$ & $\mathbf{F}$ & $p$-value \\
\hline Hip: Adduction/abduction parameters (frontal plane) & & \multicolumn{4}{|c|}{ Means (degrees) } \\
\hline Angular change in the frontal plane during loading response & $\Delta \theta_{\mathrm{HF} 1}=\theta_{\mathrm{A}-\mathrm{T} 1}-\theta_{\mathrm{A}-\mathrm{T} 0}$ & $2.8 \pm 1.6$ & $0.99 \pm 19$ & 9.765 & .003 \\
\hline Angular change in the frontal plane around the unilateral stance end & $\Delta \theta_{\mathrm{HF} 4}=\theta_{\mathrm{A}-\mathrm{T} 3+5}-\theta_{\mathrm{A}-\mathrm{T} 3-5}$ & $-1.2 \pm 1.7$ & $-0.1 \pm 1.1$ & 7.095 & .011 \\
\hline Angular change in the frontal plane during terminal swing & $\Delta \theta_{\mathrm{HF} 6}=\theta_{\mathrm{A}-\mathrm{T} 7}-\theta_{\mathrm{A}-\mathrm{T} 6}$ & $1.5 \pm 2.5$ & $0.0 \pm 1.5$ & 6.334 & .015 \\
\hline Angular change in the frontal plane during mid-stance & $\Delta \theta_{\mathrm{HF} 2}=\theta_{\mathrm{A}-\mathrm{T} 2}-\theta_{\mathrm{A}-\mathrm{T} 1}$ & $3.9 \pm 2.0$ & $4.8 \pm 2.7$ & 5.089 & .010 \\
\hline Angular change in the frontal plane during terminal stance & $\Delta \theta_{\mathrm{HF} 3}=\theta_{\mathrm{A}-\mathrm{T} 3}-\theta_{\mathrm{A}-\mathrm{T} 2}$ & $-0.04 \pm 2.0$ & $1.3 \pm 1.6$ & 5.194 & .009 \\
\hline Angular change in the frontal plane during mid-swing & $\Delta \theta_{\mathrm{HF} 5}=\theta_{\mathrm{A}-\mathrm{T} 6}-\theta_{\mathrm{A}-\mathrm{T} 5}$ & $3.4 \pm 2.4$ & $2.3 \pm 2.2$ & 4.871 & .012 \\
\hline Hip: Femoral rotation parameters (transversal plane) & & \multicolumn{4}{|c|}{ Means (degrees) } \\
\hline Mean angular position in the transverse plane during pre-swing & $\theta_{\mathrm{HT} 1}=\theta_{\text {Mean T3-T4 }}$ & $4.5 \pm 5.3$ & $2.5 \pm 6.6$ & 4.979 & .011 \\
\hline Knee: Flexion/extension parameters (sagittal plane) & & \multicolumn{4}{|c|}{ Means (degrees) } \\
\hline Angular change in the sagittal plane during loading response & $\Delta \theta_{\mathrm{KS} 1}=\theta_{\mathrm{F}-\mathrm{T} 1}-\theta_{\mathrm{F}-\mathrm{T} 0}$ & $6.2 \pm 3.4$ & $4.0 \pm 3.2$ & 3.678 & .033 \\
\hline Maximal angle in the sagittal plane during mid-stance & $\theta_{\mathrm{KS} 2}=\theta_{\mathrm{Max} \mathrm{T} 1-\mathrm{T} 2}$ & $22.4 \pm 7.6$ & $17.7 \pm 7.2$ & 6.627 & .003 \\
\hline Minimal angle in the sagittal plane during terminal stance & $\theta_{\mathrm{KS} 3}=\theta_{\text {Min T2-T3 }}$ & $9.4 \pm 7.3$ & $7.0 \pm 8.1$ & 7.504 & .002 \\
\hline Knee: Tibial rotation parameters (transversal plane) & & \multicolumn{4}{|c|}{ Means (degrees) } \\
\hline Mean angular position in the transversal plane during mid-stance & $\theta_{\mathrm{KT} 1}=\theta_{\text {Moy T1-T2 }}$ & $-0.4 \pm 3.0$ & $-0.0 \pm 3.2$ & 3.729 & .032 \\
\hline Mean angular position in the transversal plane during terminal stance & $\theta_{\mathrm{KT} 2}=\theta_{\text {Moy T2-T3 }}$ & $-0.6 \pm 2.2$ & $0.3 \pm 3.0$ & 5.180 & .010 \\
\hline Mean angular position in the transversal plane during pre-swing & $\theta_{\mathrm{KT3}}=\theta_{\text {Moy T3-T4 }}$ & $-1.7 \pm 2.5$ & $-0.6 \pm 3.0$ & 3.644 & .034 \\
\hline Ankle: Inversion/eversion parameters (frontal plane) & & \multicolumn{4}{|c|}{ Means (degrees) } \\
\hline Maximal angle in the frontal plane during gait cycle & $\theta_{\mathrm{AF} 1}=\theta_{\mathrm{Max} \mathrm{GC}}$ & $-5.1 \pm 5.9$ & $-1.3 \pm 5.4$ & 4.674 & .036 \\
\hline Angular change in the frontal plane during pre-swing & $\Delta \theta_{\mathrm{HF} 4}=\theta_{\mathrm{A}-\mathrm{T} 4}-\theta_{\mathrm{A}-\mathrm{T} 3}$ & $1.5 \pm 2.3$ & $3.1 \pm 2.3$ & 4.865 & .033 \\
\hline Mean angular position in the frontal plane during initial swing & $\theta_{\mathrm{HF} 5}=\theta_{\text {Moy T4-T5 }}$ & $-8.7 \pm 6.5$ & $-5.1 \pm 5.4$ & 4.075 & .050 \\
\hline Mean angular position in the frontal plane during mid-swing & $\theta_{\mathrm{HF6}}=\theta_{\text {Moy T5-T6 }}$ & $-9.2 \pm 6.3$ & $-5.5 \pm 5.8$ & 4.080 & .049 \\
\hline Range of motion in the frontal plane during gait cycle & $\Delta \theta_{\mathrm{HF} 2}=\theta_{\text {Max GC}}-\theta_{\text {Min GC }}$ & $9.4 \pm 2.7$ & $11.6 \pm 2.7$ & 5.707 & .006 \\
\hline Angular change in the frontal plane during loading response & $\Delta \theta_{\mathrm{HF} 3}=\theta_{\mathrm{A}-\mathrm{T} 1}-\theta_{\mathrm{A}-\mathrm{T} 0}$ & $-4.5 \pm 1.9$ & $-5.8 \pm 3.0$ & 6.290 & .004 \\
\hline Ankle: Dorsi/plantaflexion parameters (sagittal plane) & & \multicolumn{4}{|c|}{ Means (degrees) } \\
\hline Maximal angle in the sagittal plane during loading response & $\theta_{\mathrm{AS} 1}=\theta_{\mathrm{Max} \mathrm{T} 0-\mathrm{T} 1}$ & $6.3 \pm 4.4$ & $8.4 \pm 3.8$ & 5.820 & .006 \\
\hline Mean angular position in the sagittal plane during mid-stance & $\theta_{\mathrm{AS} 2}=\theta_{\text {Moy T1-T2 }}$ & $-1.9 \pm 3.9$ & $0.3 \pm 4.5$ & 5.347 & .008 \\
\hline Mean angular position in the sagittal plane during terminal stance & $\theta_{\mathrm{AS} 3}=\theta_{\mathrm{Moy} \mathrm{T2-T3}}$ & $-10.5 \pm 3.9$ & $-8.5 \pm 4.2$ & 5.378 & .008 \\
\hline Angular change in the sagittal plane during initial swing & $\Delta \theta_{\mathrm{AS} 4}=\theta_{\mathrm{A}-\mathrm{T} 5}-\theta_{\mathrm{A}-\mathrm{T} 4}$ & $9.6 \pm 3.9$ & $12.7 \pm 6.0$ & 3.625 & .035 \\
\hline Maximal angle in the sagittal plane during swing & $\theta_{\mathrm{AS} 5}=\theta_{\mathrm{Max} \text { T4-T7 }}$ & $7.3 \pm 5.5$ & $10.4 \pm 6.0$ & 6.623 & .003 \\
\hline Minimal angle in the sagittal plane during swing & $\theta_{\mathrm{AS} 6}=\theta_{\mathrm{Min} \mathrm{T} 5-\mathrm{T} 7}$ & $-4.6 \pm 3.6$ & $-1.8 \pm 4.1$ & 7.515 & .002 \\
\hline
\end{tabular}

At the knee, in the frontal plane, although there was a trend towards more knee adduction (varus) throughout the gait cycle for OA patients, this was not statistically significant. Mean varus thrust in OA patients also tended to be more important, but was not significant. In the sagittal plane, OA patients flexed less their knee during stance phase. Angular change during loading response was smaller than for AS

Published by Sciedu Press

$\left(\Delta \theta_{K S 1}\right)$. Maximal and minimal angles during stance phase were lower $\left(\theta_{K S 2}\right.$ and $\left.\Delta \theta_{K S 3}\right)$ with 4.7 and 2.4 degrees less on average. At last, in the transverse plane, OA subjects remained more in internal tibial rotation compared to AS during mid-stance, terminal stance and pre-swing $\left(\theta_{K T 1}, \theta_{K T 2}\right.$, $\left.\theta_{K T 3}\right)$

Finally, the ankle was less in eversion for OA compared to 
AS. In fact, the maximal angle reached in the frontal plan during the gait cycle was greater, i.e. less in eversion, for OA $\left(\theta_{H F 1}\right)$ and the total range of motion was more important for OA compared to AS $\left(\Delta \theta_{H F 2}\right)$. Furthermore, at loading response, the ankle performed a rapid eversion for AS but even more for OA $\left(\Delta \theta_{H F 3}\right)$. During pre-swing, OA participants executed an inversion movement in average twice bigger than AS ones $\left(\Delta \theta_{H F 4}\right)$. This was followed by a mean angular position much less in eversion $\left(\theta_{H F 5}\right.$ and $\left.\theta_{H F 6}\right)$ for OA. In the sagittal plane, ankle was generally more in plantar flexion or less in dorsiflexion for OA compared to AS. This was significant during changes in direction of movement $\left(\theta_{A S 1}\right.$, $\theta_{A S 5}$ and $\theta_{A S 6}$ ) on mean angular position during unilateral stance $\left(\theta_{A S 2}\right.$ and $\left.\theta_{A S 3}\right)$ and on angular change during initial swing $\left(\Delta \theta_{A S 4}\right)$. In the transverse plane, none of the results were significant.
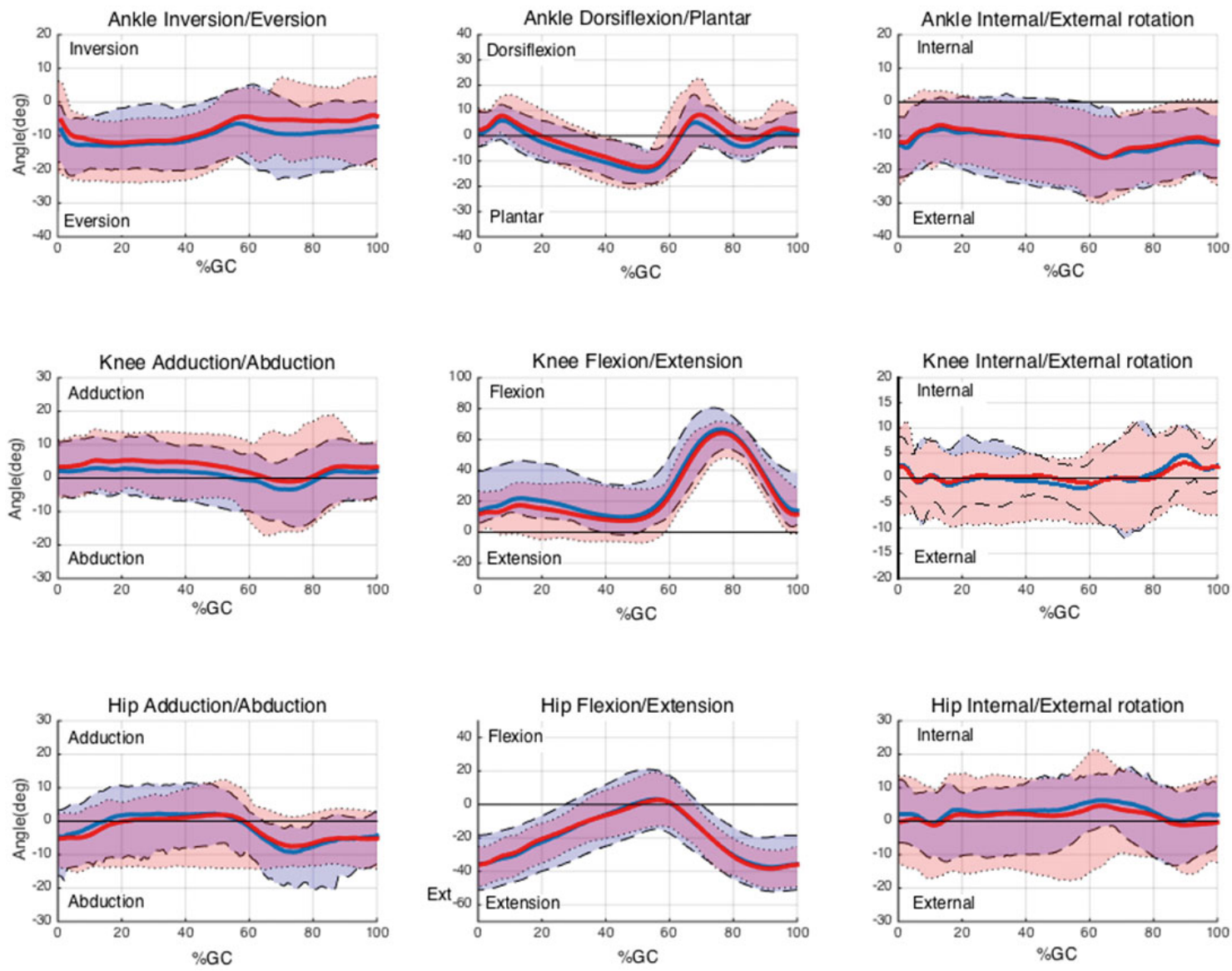

Figure 1. Angular patterns of the displacement of the Ankle, knee and hip joints (rows), in frontal, sagittal and transversal plans (columns), during a gait cycle. The blue and red lines represent respectively the AS and OA groups average patterns.

\section{Discussion}

3D kinematic patterns of OA gait revealed significant differences with those of AS subjects at the three joints of lower limb. The presence of osteoarthritis at the knee, therefore, led to adjustments of angular displacement at the knee, but also of the hip and ankle.

In agreement with other studies, ${ }^{[21-24]}$ results show a decreased stance phase knee flexion for OA patients. ${ }^{[22]}$ However, a decreased peak knee flexion during swing phase was not observed in our cohort of patients. Moreover, during loading response and unilateral stance, while OA participants flexed less their knee, their ankle was first more in plantar flexion. As the gait cycle progressed, it was less in dorsiflexion. We can assume that since OA minimize their knee flexion, ankle dorsiflexion is also logically minimized when the body weight crosses the vertical line on the evaluated leg.

When focusing on the first 10 percent of the gait cycle, a precautionary loading response seems to appear in OA patients: at the hip, abduction is achieved significantly later and more slowly than AS subjects; in addition the knee is 
much less flexed; finally, the ankle performs a reduced eversion movement and an increased plantar flexion. Overall, all these indications appear as an adaptive strategy, likely to increase knee stability and to optimize propulsion. It has been described in an even more extended way by Bytyqi et al. (2014) in OA patients waiting for a total knee arthroplasty (mostly grade III and IV) and characterized as an "altered screw-home mechanism by decreased excursion in sagittal and axial tibial rotation". In their study, more important differences were found for the knee in the frontal plane. This can be explained by the fact that the patients in the present study had generally lower grades of OA, compared to patients in Bytyqi et al.'s study. ${ }^{[12]}$

During push-off phase of the gait cycle, results show increased ankle rotations in the frontal and sagittal plane. This support previous findings from Ko, et al. (2011) ${ }^{[10]}$ which hypothesized that this increase in sagittal range of motion is a compensatory mechanism in symptomatic knee-OA patient during propulsion to limit the demand on the knee joint to limit onset of symptoms.

Kinematic adaptations of lower limb joints of patients with hip osteoarthritis have been described in. ${ }^{[25]}$ These patients walk differs from controls including a reduction of maximum hip flexion and extension and an increase of ankle dorsiflexion. In the present case of knee OA subjects, the hip kinematics in the sagittal plane is very similar to asymptomatic participant one. Their ankle, as stated earlier, is on the contrary rather more in plantar flexion during walking. Therefore, it seems that compensatory strategies could be different between these two diseases.

\section{Conclusion}

3D kinematic gait patterns of OA patients reveal significant differences with those of AS subjects at the three joints of the lower limb. The presence of knee osteoarthritis leads to adjustment of angular displacement's strategies at the knee, but also at the hip and ankle.
This study has some limitations including mainly the lack of radiological verification of the absence of hip and ankle osteoarthritis: the subjects were not specifically tested at this level since original data acquisition found interest in knee only. However, generalized osteoarthritis or rheumatoid arthritis was an exclusion criterion and all subjects were clinically evaluated by an experienced physiatrist who ruled out all patients that might present arthritis at multiple joints.

The interest of this project lies in the study of the three lower limb joints kinematics for the same subjects, and not only the one affected by the pathology. The statistical analysis shows that hip and ankle also experience adaptations of their biomechanics in presence of osteoarthritis at the knee; this synergy motivates the consideration of care of knee OA by treating also the other joints, as for example encouraged by Thorp, et al. ${ }^{[7]}$ Their study suggests targeting the hip. Indeed, focusing on hip abductor muscles training may represent an effective option to reduce external adduction knee moment and the knee pain score of patients. The same year, a randomized study of Bennell, et al. (2010) ${ }^{[8]}$ confirms that strengthening the hip muscles improves symptoms and knee OA function but does not affect the medial knee loading measured by the adduction moment, nor does it affect the progression of the pathology. Research is still necessary to develop optimal physiotherapy programs for knee OA patients.

To conclude, this study opens the way for further research to identify coping strategies of the locomotor system against disease. Dynamic mechanical biomarkers assess through a biomechanical assessment could help tailor the care pathway and better design treatment protocols.

\section{ACKNOWLedgements}

The authors would like to thank the Programme MITACS of Fonds québécois de la recherche sur la nature et les technologies (FQRNT) and the Natural Sciences and Engineering Research Council (NSERC). Special thanks to Gerald Parent for technical support and Karine Boivin for her help in data collection.

\section{REFERENCES}

[1] Woolf AD, Pfleger B. Burden of major musculoskeletal conditions. Bull World Health Organ. 2003; 81(9): 646-56. PMid:14710506.

[2] Andriacchi TP, Koo S, Scanlan SF. Gait mechanics influence healthy cartilage morphology and osteoarthritis of the knee. J Bone Joint Surg Am. 2009; 91 Suppl 1: 95-101. https://doi.org/10. 210 6/JBJS.H.01408

[3] Hunter DJ. Focusing osteoarthritis management on modifiable risk factors and future therapeutic prospects. Ther Adv Musculoskelet Dis. 2009; 1(1): 35-47. PMid:22870426. https ://doi .org/10.1 $177 / 1759720 \times 09342132$
[4] Gaudreault N, et al. Effects of physiotherapy treatment on knee osteoarthritis gait data using principal component analysis. Clin Biomech (Bristol, Avon). 2011; 26(3): 284-91. PMid:21071119. https://doi.org/10.1016/j.clinbiomech.2010.10.004

[5] Omori G. Association of mechanical factors with medial knee osteoarthritis: A cross-sectional study from Matsudai Knee Osteoarthritis Survey. Osteoarthr Cartil. 2016; 24: S221. https ://doi.org/ $10.1016 / j \cdot$ joca. 2016.01.425

[6] Bytyqi D, et al. Gait knee kinematic alterations in medial osteoarthritis: three dimensional assessment. Int Orthop. 2014.

[7] Thorp LE, et al. The biomechanical effects of focused muscle training 
on medial knee loads in OA of the knee: a pilot, proof of concept study. J Musculoskelet Neuronal Interact. 2010; 10(2): 166-73.

[8] Bennell KL, et al. Hip strengthening reduces symptoms but not knee load in people with medial knee osteoarthritis and varus malalignment: a randomised controlled trial, in Osteoarthritis Cartilage. England. 2010. p. 621-8.

[9] Astephen JL, et al. Gait and neuromuscular pattern changes are associated with differences in knee osteoarthritis severity levels. J Biomech. 2008; 41(4): 868-76. PMid:18078943. https://doi.or $\mathrm{g} / 10.1016 / \mathrm{j} \cdot \mathrm{jbiomech} \cdot 2007.10 .016$

[10] Ko SU, et al. Gait patterns during different walking conditions in older adults with and without knee osteoarthritis-results from the Baltimore Longitudinal Study of Aging, in Gait Posture. England. 2011. p. 205-10.

[11] Farrokhi S, et al. A biomechanical perspective on physical therapy management of knee osteoarthritis. J Orthop Sports Phys Ther. 2013; 43(9): 600-19. PMid:23756435. https://doi.org/10.2519/jo spt. 2013.4121

[12] Bytyqi D, et al. Gait knee kinematic alterations in medial osteoarthritis: three dimensional assessment. Int Orthop. 2014; 38(6): 11918. PMid:24619388. https://doi.org/10.1007/s00264-014-2 312-3

[13] Boivin K. Développement d'une approche d'évaluation clinique de la cinématique tridimensionnelle du genou durant la marche pour des patients gonarthrosiques. 2010. p. 299.

[14] Turcot K. Développement d'une méthode accélérométrique tridimensionnelle pour l'évaluation d'une population atteinte d'arthrose du genou: application à un contexte de marche. 2008 .

[15] Hagemeister N, et al. A reproducible method for studying threedimensional knee kinematics. Journal of Biomechanics. 2005 September; 38(9): 1926-31.

[16] Lustig S, et al. The KneeKG system: a review of the literature. Knee Surgery, Sports Traumatology, Arthroscopy. 2012; 20(4): 633638. PMid:22215077. https://doi .org/10.1007/s00167-011 $-1867-4$
[17] Mechmeche I, Mitiche A, Ouakrim Y, et al. Data correction to determine a representative pattern of a set of 3D knee kinematic measurements. EMBC. 2016: 884-887.

[18] Mezghani N, Ouakrim Y, Fuentes A, et al. Severity grading mechanical biomarkers of knee osteoarthritis. Ostearthritis and Cartilage. Avril 2016; 24: S125-S126.

[19] Mezghani N, Ouakrim Y, Fuentes A, et al. Mechanical biomarkers of medial compartment knee osteoarthritis diagnosis and severity grading: Discovery phase. Journal of Biomechanics. 2017; 52: 106-112. PMid:28088304. https://doi.org/10.1016/j.jbiomech. 201 6.12 .022

[20] Labbe DR, et al. Reliability of a method for analyzing threedimensional knee kinematics during gait. Gait Posture. 2008; 28(1): 170-4. PMid:18155553. https://doi.org/10.1016/j.gaitpo st. 2007.11 .002

[21] Al-Zahrani KS, Bakheit AM. A study of the gait characteristics of patients with chronic osteoarthritis of the knee. Disabil Rehabil. 2002; 24(5): 275-80. https ://doi.org/10.1080/0963828011 0087098

[22] Astephen JL, et al. Biomechanical changes at the hip, knee, and ankle joints during gait are associated with knee osteoarthritis severity. Journal of Orthopaedic Research: official publication of the Orthopaedic Research Society. 2008; 26(3): 332-41. PMid:17960658. https://doi.org/10.1002/jor. 20496

[23] Kaufman KR, et al. Gait characteristics of patients with knee osteoarthritis. Journal of Biomechanics. 2001; 34(7): 907-15. https : //doi.org/10.1016/S0021-9290(01) 00036-7

[24] Gok H, Ergin S, Yavuzer G. Kinetic and kinematic characteristics of gait in patients with medial knee arthrosis. Acta Orthop Scand. 2002; 73(6): 647-52. https://doi.org/10.3109/1745367020 9178029

[25] Ornetti P, et al. Three-dimensional kinematics of the lower limbs in hip osteoarthritis during walking, in J Back Musculoskelet Rehabil. Netherlands. 2011. p. 201-8. 